\title{
A New Soft-Tissue Simulation Strategy for Cranio-Maxillofacial Surgery Using Facial Muscle Template Model
}

\author{
Hyungmin Kim ${ }^{\mathrm{a}, *}$, Philipp Juergens ${ }^{\mathrm{b}}$, Stefan Weber $^{\mathrm{c}}$, Lutz-Peter Nolte ${ }^{\mathrm{a}}$, \\ Mauricio Reyes ${ }^{\mathrm{a}}$ \\ ${ }^{a}$ Institute for Surgical Technology and Biomechanics, University of Bern, \\ Stauffacherstrasse 78, 3014 Bern, Switzerland \\ ${ }^{b}$ Department of Cranio-Maxillofacial Surgery, University Hospital Basel, Spitalstrasse \\ 21, 4031 Basel, Switzerland \\ ${ }^{c}$ ARTORG Center for Biomedical Engineering Research, University of Bern, \\ Stauffacherstrasse 78, 3014 Bern, Switzerland
}

\begin{abstract}
We propose a computationally efficient, bio-mechanically relevant soft-tissue simulation method for cranio-maxillofacial (CMF) surgery. Special emphasis is given to comply with the current clinical workflow. A template-based facial muscle prediction was introduced to avoid laborious segmentation from medical images. In addition, transversely isotropic mass-tensor model (MTM) was applied to realize the directional behavior of facial muscles in short computation time. Finally, sliding contact was incorporated to mimic realistic boundary condition in error-sensitive regions. Mechanical simulation result was compared with commercial finite element software. And retrospective validation study with post-operative scan of four CMF cases was performed.
\end{abstract} Keywords: soft-tissue simulation, mass-tensor model, cranio-maxillofacial

\footnotetext{
*Corresponding author. Tel: +41 (0)31 631 5952; Fax: +41 (0)31631 5960

Email address: hyungmin.kim@istb.unibe.ch (Hyungmin Kim)
} 
surgery

\section{Introduction}

Cranio-maxillofacial (CMF) surgery is a surgical procedure, which corrects facial bone deformity to improve functional and aesthetic outcome. Computer-assisted surgery (CAS) has been widely applied in this field, and successfully integrated into the clinical workflow. Three-dimensional imaging and rapid-prototyping technique help surgeons to plan the operation both on virtual and tangible models, whereas optical tracking technology enables the intra-operative guidance in the operating theater for various CMF applications (Hallermann et al., 2006; Chapuis et al., 2007). Along with the successful applications on rigid anatomical parts, there have been increasing demands on predicting soft-tissue changes after CMF surgeries. Some of them have been realized as commercial surgical simulation softwares.

There have been many studies on facial soft-tissue modeling from different fields of research. They can be classified into three categories depending on the computational method: mass-spring model (MSM), finite-element model (FEM), and mass-tensor model (MTM). First, MSM was initially accepted for real-time face animation due to its well-known physics and computational efficiency. Terzopoulos and Waters (1990) and Lee et al. (1995) proposed a fast simulation method using MSM with linear facial muscle model. Keeve et al. (1998) presented a MSM with prismatic element, and compared the result with FEM in terms of accuracy and computational cost. Teschner et al. (2000) proposed a multi-layer non-linear MSM with a static constraint to directly compute equilibrium state. Second, FEM has strong bio-mechanical 
relevance at the cost of long calculation time. Koch et al. (1996) proposed a non-linear finite-element surface model with linear springs attached to the bone. Sarti et al. (1999) presented a computationally expensive voxel-based FEM running on a super computer. Zachow et al. (2000) suggested a fast tetrahedral volumetric FEM, which can be applicable to clinical practice. Later, Gladilin (2003) extended this to functional simulation with facial muscles. Chabanas et al. (2003) proposed a mesh morphing algorithm to minimize the laborious efforts on preparing finite-element mesh. Third, MTM was originally developed to find a golden mean between speed and accuracy. Cotin et al. (2000) proposed a hybrid method of using MTM for local deformations. Picinbono et al. (2003) extended this approach to non-linear, anisotropic elasticity. Mollemans et al. (2007) first applied MTM to CMF soft-tissue simulation, and the method was validated the results with 10 clinical cases both in quantitative and qualitative aspects. Roose et al. (2006) applied MTM to breast implant augmentation simulation with consideration on various boundary conditions.

Even though comprehensive amount of studies have been performed, most of the previous works have failed to be seamlessly integrated into the clinical workflow in two aspects. On the one hand, due to the natural higher perceptibility of subtle changes on the human face, the simulation accuracy provided by current solutions is not sufficient for CMF surgeons. For instance, existing solutions fail to realize delicate soft-tissue variations around nose and lips area commonly produced by underlying jaw movements. On the other hand, more sophisticated method, such as finite-element model, requires considerable pre-processing work and computation time. All these 
factors have shown to hinder the usability and acceptance of available commercial and academic solutions into the clinical routine.

In this study, we propose a method for accurate yet computationally efficient simulation of soft tissue deformation after CMF surgery, while respecting the current clinical workflow. To achieve higher accuracy, we hypothesize that patient-specific muscle characterization is necessary. To avoid the daunting task of segmenting muscle tissue from medical images, we propose an efficient way to incorporate patient-specific facial muscles utilizing template model. We adopted transversely isotropic MTM for efficient calculation and incorporation of muscle fiber directions. From our recent promising result with single case (Kim et al., 2010), sliding contact around teeth and mucosa area was adopted for enhancing the anatomical reality on these error-prone regions. We also performed the sensitivity analysis on the simulation results by varying anatomical landmark definition in muscle morphing procedure. The proposed method is compared with FEM simulation in terms of computation time, and retrospectively validated with four post-operative CT scans for accuracy analysis.

\section{Methods}

The overview of our simulation pipeline is depicted in Figure 1. Firstly, image segmentation of bone tissues and outer skin layer is performed on conventional Computed Tomography (CT) of the patient without introducing any additional imaging modality such as Magnetic Resonance Imaging (MRI). Secondly, surface and volumetric meshes are generated from segmented masks. Bone segments are prepared by osteotomy planning software, 
then corresponding boundary conditions can be defined. Thirdly, patientspecific muscles are reconstructed from the facial template model using an elastic deformation model. The directional property of muscles is then extracted and equivalent material properties are assigned. Finally, numerical simulation with mass-tensor model is performed. Detailed explanations of each procedure are presented in next subsections.

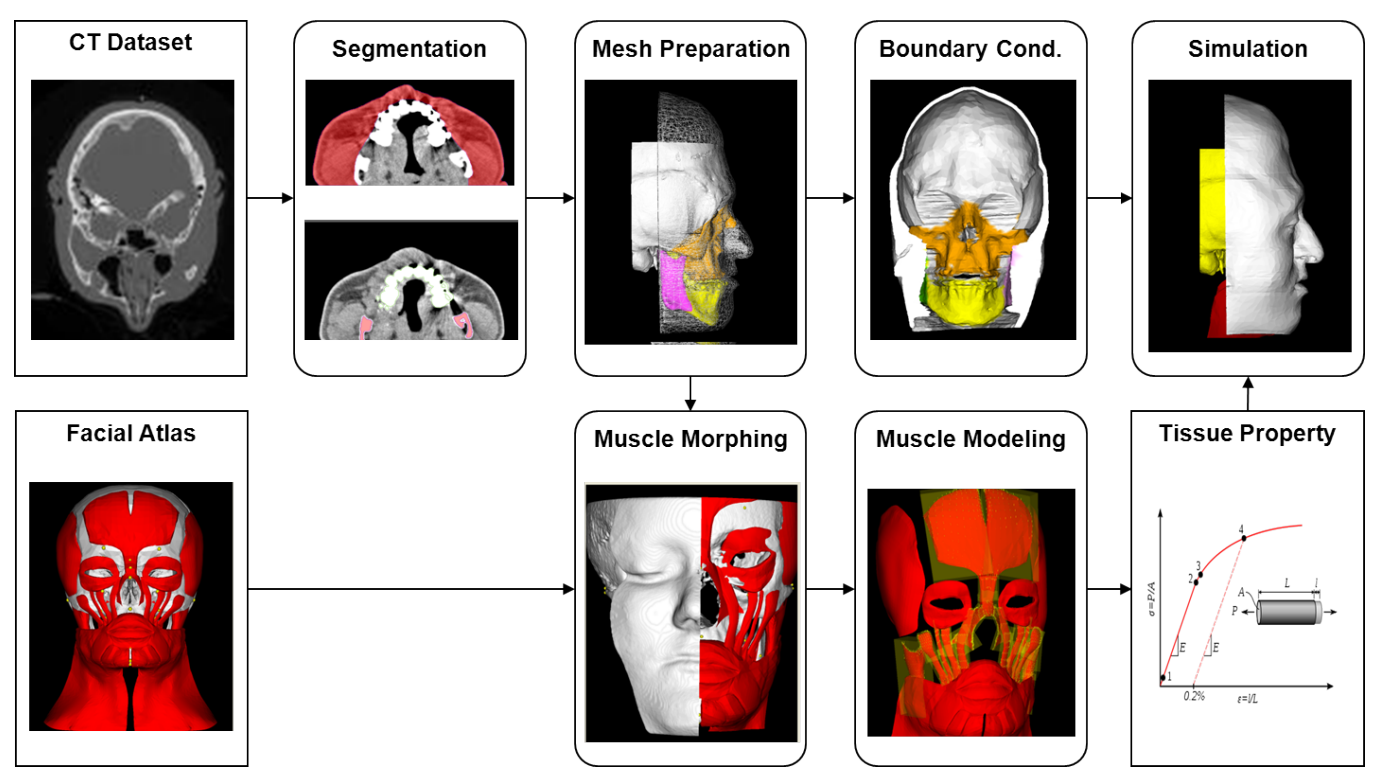

Figure 1: The pipeline of the anatomically considered facial soft-tissue simulation method

\subsection{Extra-cranial soft-tissue modeling}

Amira software (Mercury Computer Systems, Germany) was used for segmenting extra-cranial soft-tissue from clinical CT scan. We assumed that only frontal extra-cranial soft-tissue was mainly engaged in deformation of most jaw movements, which enables us to minimize computation time without losing generality. Semi-automatic segmentation tools in Amira such as 
lasso or magic wand were effectively used to accelerate segmentation procedures. Based on this segmentation result, surface mesh was generated using conventional marching cube algorithm (Lorensen and Cline, 1987), followed by aumomatized tetrahedral volumetric mesh generation.

\subsection{Patient-specific muscle modeling}

Since it is almost impossible to identify individual muscles from clinical CT scan, we propose to construct patient-specific facial muscles by morphing muscles from facial template model. We found out one virtual atlas model, which was developed by Smith et al. (2007) for educational purposes, and freely available online. The morphing procedure was driven by landmarkbased thin-plate-spline (TPS) deformation (Bookstein, 1989). We adopted 32 anatomical landmarks, which are commonly used for measuring skin depth in forensic science (Phillips and Smuts, 1996).
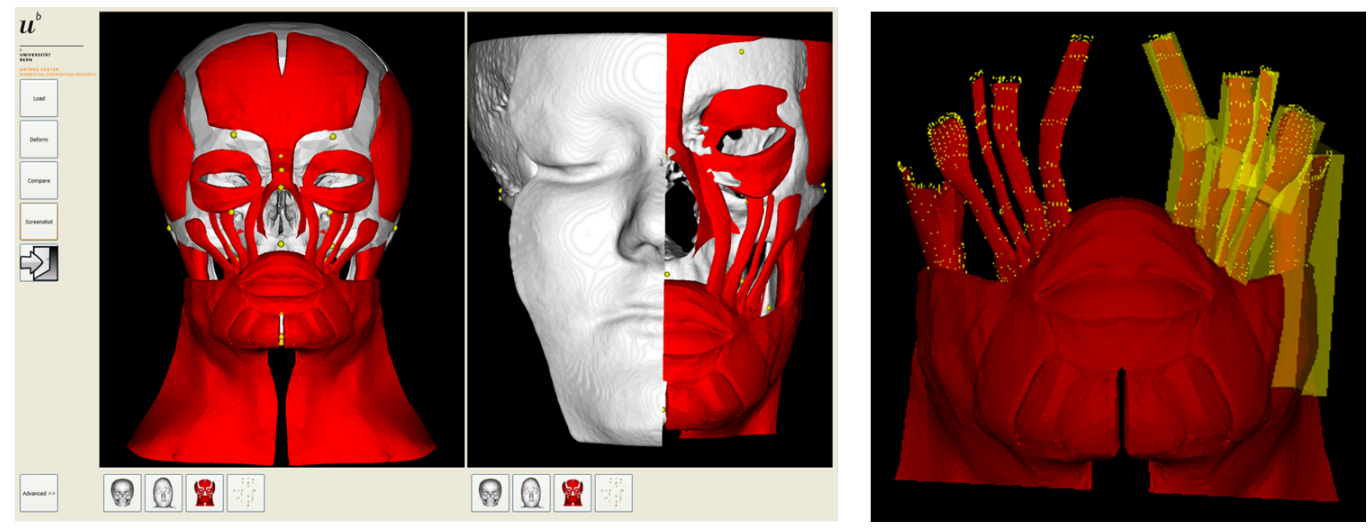

Figure 2: Morphing of template muscles to patient-specific anatomy (left), and extraction of morphed muscle direction using oriented-bounding boxes (right)

In order to obtain the direction of muscles, oriented-bounding boxes 
(OBB) were extracted for linear-type muscles. As shown in Figure 2, the number of OBBs for individual muscle can be adjusted according to the curvature of the muscle. For example, masseter was represented by single OBB, whlie the other muscles were represented by double OBBs. The longitudinal direction of each bounding box was regarded as the direction of muscle for each segment.

\subsection{Material property assignment}

As far as we investigated, there was no consensus in the literature on mechanical properties of facial soft-tissue. Different material parameters were given by the previous works depending on characterization method and material model. We adopted Young's modulus of muscle tissue along and across fiber direction from previous study (Duck, 1990). We also referred to the studies on estimating optimal facial tissue properties by comparing their simulation models with real post-operative data (Zachow et al., 2004; Mollemans et al., 2006). The parameters that we selected for our simulation are shown in Table 1. Since there was no previous study found on anisotropic Poisson ratio of muscle, we had to assume isotoropic Poisson ratio.

Table 1: Material properties used for simulation.

\begin{tabular}{ccc}
\hline & Young's modulus (MPa) & Poisson Ratio \\
\hline Fat & 0.003 & 0.46 \\
Muscle across fiber & 0.79 & 0.43 \\
Muscle along fiber & 0.5 & 0.43 \\
\hline
\end{tabular}

Since it is difficult to remesh volumetric facial tissue according to the 
intersection with muscle surfaces, we calculated equivalent material property by considering volumetric portion of muscle in each tetrahedron. We adopted the approach proposed by Uesu et al. (2005), which employs point sampling in tetrahedron. Then, geometrical test, based on directional comparison of surface normal and closest distance vector as shown in Figure 3, was performed to calculate equivalent material properties.

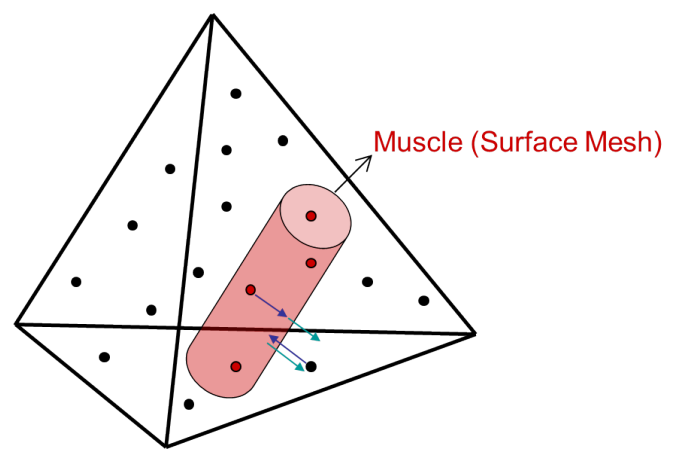

Figure 3: Calculation of intersecting portion of muscle in each tetrahedron. Random points (black dots) are generated inside a tetrahedron and geometrical tests, described by the arrows, allow definition of material properties.

The equivalent material properties can be define by the volumetric portion of muscle $\left(p_{\text {muscle }}\right)$ in each tetrahedron.

$$
\begin{aligned}
E & =E_{\text {fat }} *\left(1-p_{\text {muscle }}\right)+E_{\text {muscle }} * p_{\text {muscle }} \\
\nu & =\nu_{\text {fat }} *\left(1-p_{\text {muscle }}\right)+\nu_{\text {muscle }} * p_{\text {muscle }}
\end{aligned}
$$

\subsection{Mass-tensor modeling}

MTM can be regarded as a trade-off solution between MSM and FEM. It has bio-mechanical relevance as FEM, while maintaining the computational 
efficiency as MSM. We refered to MTM formulations of the works of Cotin et al. (2000), Picinbono et al. (2003) and Mollemans et al. (2007), where the reader can find further details.

In MTM formulation, the internal force on the $\mathrm{j}$-th node of the volumetric mesh can be expressed as

$$
\mathbf{f}_{j}=\mathbf{K}_{j j} \mathbf{u}_{j}+\sum_{\forall k \in \Psi_{j}} \mathbf{K}_{j k} \mathbf{u}_{k}
$$

where $\Psi_{j}$ is the group of vertices connected to the $\mathbf{j}$-th node, $\mathbf{u}_{j}$ is the displacement vector on $\mathbf{j}$-th node. $\mathbf{K}_{j j}$ and $\mathbf{K}_{j k}$ are the stiffness tensors for vertex and edge respectively, which are only dependent on material properties and initial mesh configuration.

\subsection{Boundary condition assignment}

As shown in Figure 4, the volumetric nodes were classified into four types : fixed, joined, slide and free nodes. We defined fixed nodes along the most posterior plane of volumetric soft-tissue model, which can be assumed to be fixed during the simulation. Joined nodes were defined on the interface between relocated bone segments and soft-tissue. Additionally, we defined sliding nodes on the interface around the teeth and mucosa area.

On sliding nodes, only the tangential component to closest contacting triangle will affect the movement along the contacting surface. Neglecting frictional forces, the tangential force $\mathbf{f}_{j}^{t}$ can be calculated by

$$
\mathbf{f}_{j}^{t}=\mathbf{f}_{j}-\left(\mathbf{f}_{j} \cdot \mathbf{n}_{p}\right) \mathbf{n}_{p}
$$

where $\mathbf{f}_{j}$ is the nodal force given by Eq.1, and $\mathbf{n}_{p}$ is the normal vector of nearest contacting triangle. 

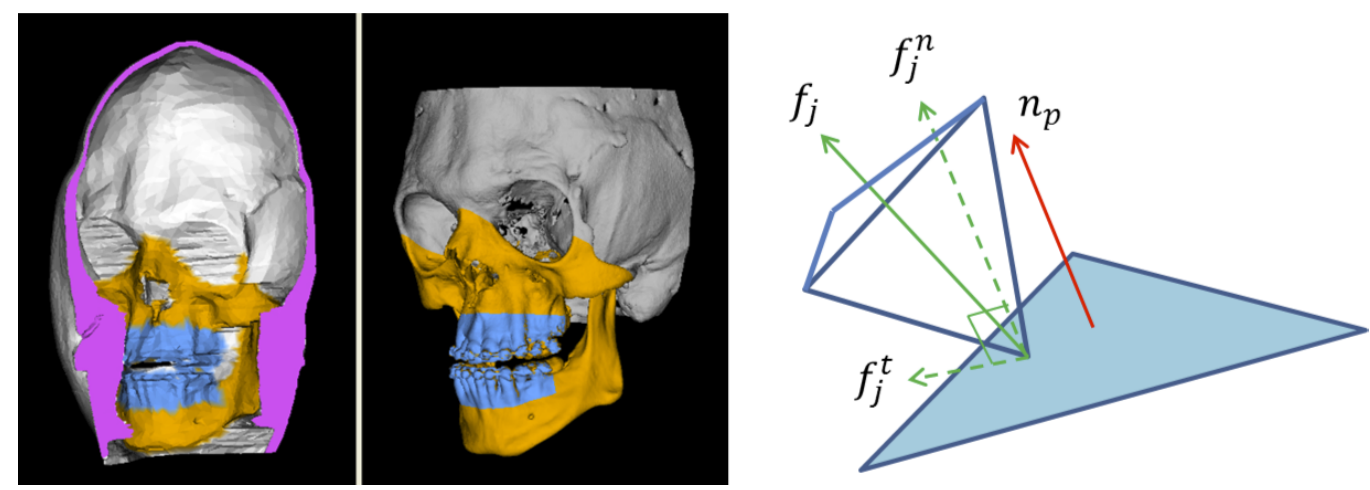

Figure 4: Classification of volumetric nodes: fixed(pink), joined(orange), sliding(blue) and free(white) (left), force diagram on sliding contact surface (right)

\section{Results}

The retrospective validation study was performed with four clinical cases. The first patient was suffered from a craniofacial deformation named PfeifferSyndrom, which showed a mid-facial hypoplasia as leading clinical sign. The second patient was suffering from an Angle class III malocclusion with a maxillary retrognatism and a mandibular prognatism, and was treated with a bimaxillary procedure including genioplastia. The third patient also presented a class III malocclusion accompanied by a laterognatism to the right side. The fourth patient was treated because of a mandibular retrognatism, a long face syndrome and an open bite. These patients were chosen from the database, because they can represent nearly all possible variations of skeletal malocclusions. Details on the patients and the performed movements in the maxilla are presented in Table 2.

The actual displacement of bone segments during the operation has to be reproduced by matching the pre-operative and post-operative images. First, 
Table 2: Surgical procedures performed on patient cases

\begin{tabular}{ccl}
\hline Dataset & Surgical Procedure & $\begin{array}{l}\text { Maxillary Movement }[\mathrm{mm}, \mathrm{mm}, \mathrm{mm}, \text { degree }] \\
\left(\mathrm{Tx}, \mathrm{Ty}, \mathrm{Tz}, R_{\text {arbitrary }}\right)\end{array}$ \\
\hline P1 & $\mathrm{MxA}$ & $(0.53,-11.58,-3.78,3.39)$ \\
P2 & $\mathrm{MSB}+\mathrm{G}$ & $(-3.24,-0.61,0.67,0.00)$ \\
P3 & $\mathrm{MxA}+\mathrm{G}$ & $(0.27,-4.98,-0.13,1.31)$ \\
P4 & $\mathrm{MxA}+\mathrm{MA}+\mathrm{G}$ & $(1.07,-5.47,0.74,2.71)$ \\
\hline
\end{tabular}

MA: Mandibular Advancement MSB: Mandibular Setback

MxA: Maxillary Advancement MxSB: Maxillary Setback

G: Genioplasty

the post-operative data was aligned with pre-operative data by using volumetric image registration method on undeformed skull part, as shown in Figure 5(left). Next, the actual bone-related planning was reproduced by performing ICP-based registration between pre- and post-operative bone segments, as shown in Figure 5(right) (Besl and McKay, 1992). The reproduced relocation planning is depicted in Figure 6.

Especially in patient case P1, we used an in-house developed navigation system (Chapuis et al., 2007) to control the distraction vector for the midfacial advancement as shown in Figure 7. By means of computer-assisted planning, a two-step procedure was identified to be the ideal treatment for that patient. In step one, a Le-Fort III osteotmy with osteodistraction of the facial complex was planned as shown in Figure 6(P1). In step two, bimaxillary osteotomy with monobloc advancement was considered. 

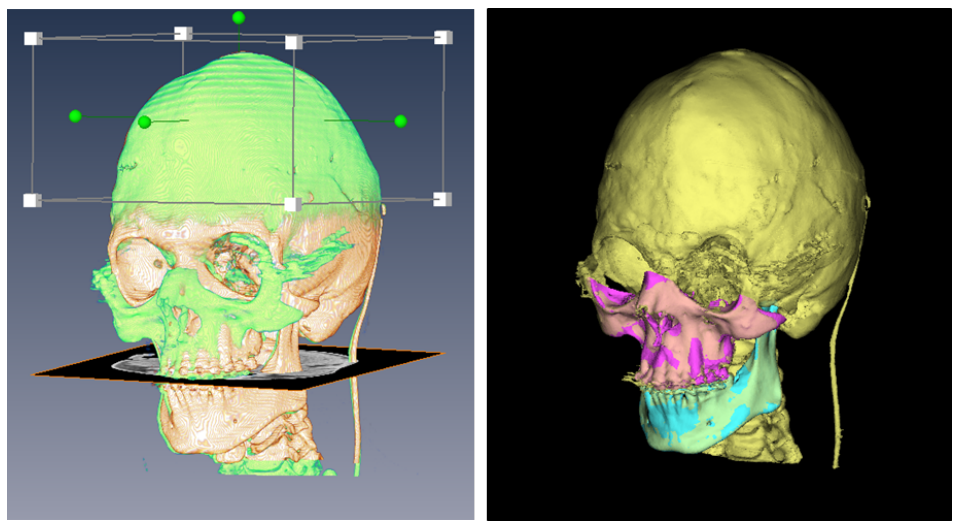

Figure 5: Reproduction of bone-related planning for retrospective validation study: registration of pre- and post-operative images (left), registration of pre-operative bone segments to post-operative yellow skull model (right).

\subsection{Comparison with finite-element simulation}

As previously mentioned, MTM was developed to provide a golden mean between accuracy and computation time. We were interested in comparing the computational performance of our MTM implementation with commercial FEM simulation using ABAQUS/CAE 6.7 (Dassault Systems, RI, USA). In this study, we only used patient case 1 data with same elements and boundary conditions for both simulation methods. The differences between FEM and MTM simulation was measured as $0.023 \mathrm{~mm} \pm 0.0061 \mathrm{~mm}$. The overall computation of MTM was almost 18 times faster than FEM (MTM: $3.8 \mathrm{sec}$, FEM: $67 \mathrm{sec}$ ). The computational efficiency can be even higher by considering net computation time of MTM (1.3 sec), since stiffness tensor matrices of MTM can be pre-computed and applied to various surgical plans without recalculation. This computational superiority can be maintained when it is extended to transversely isotropic model. We emphasize that the goal of this 


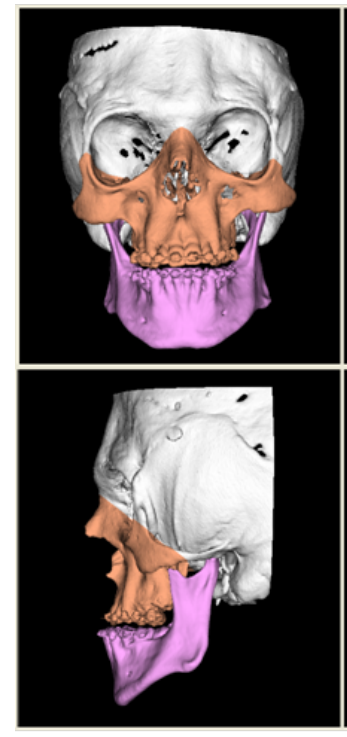

Pre-op

P1
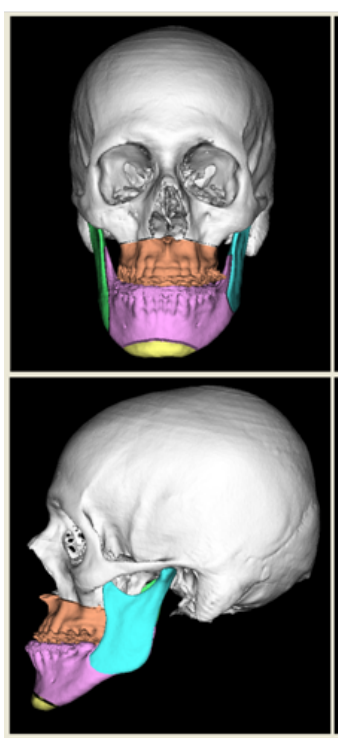

Pre-op
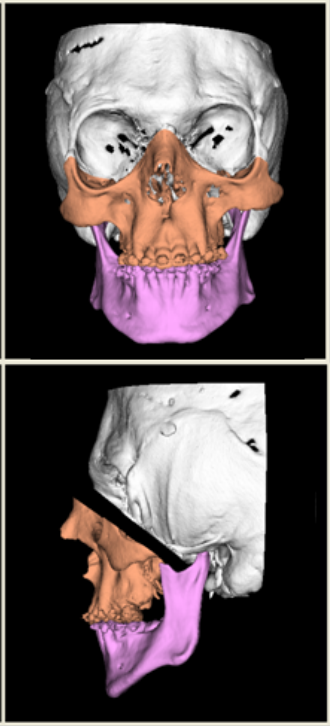

Post-op
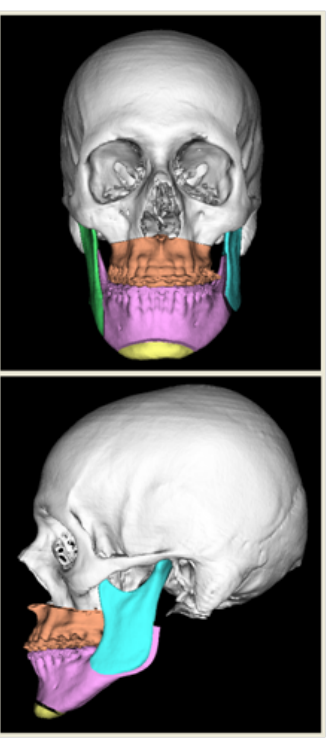

P3
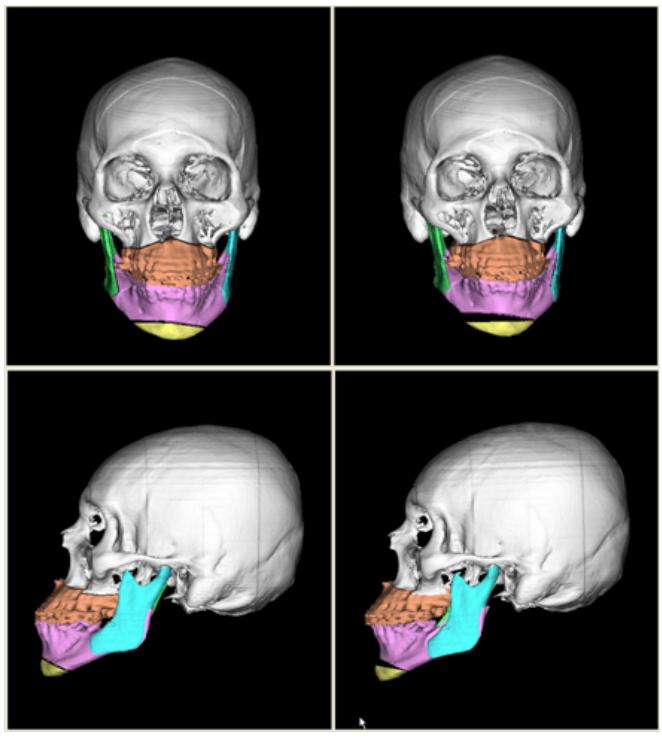

Pre-op

P2
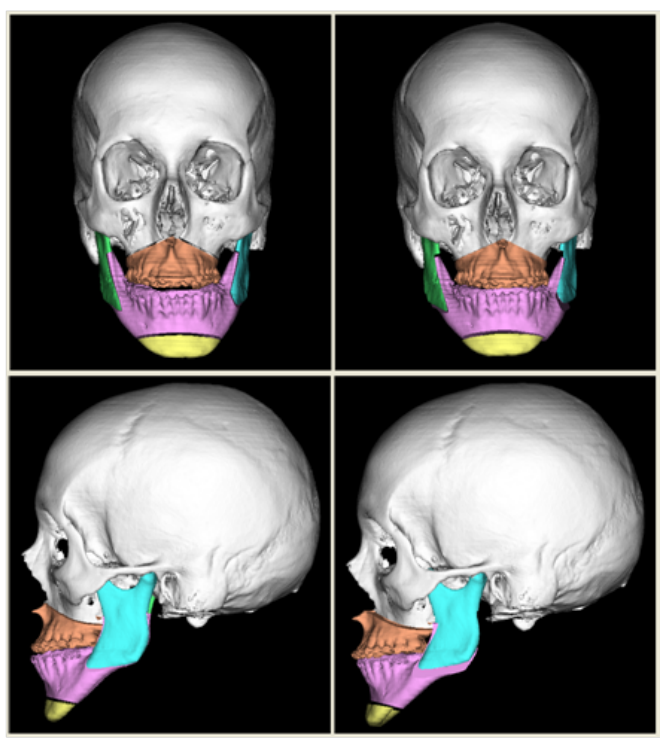

Pre-op

P4

Figure 6: Reproduced relocation planning of four clinical cases 

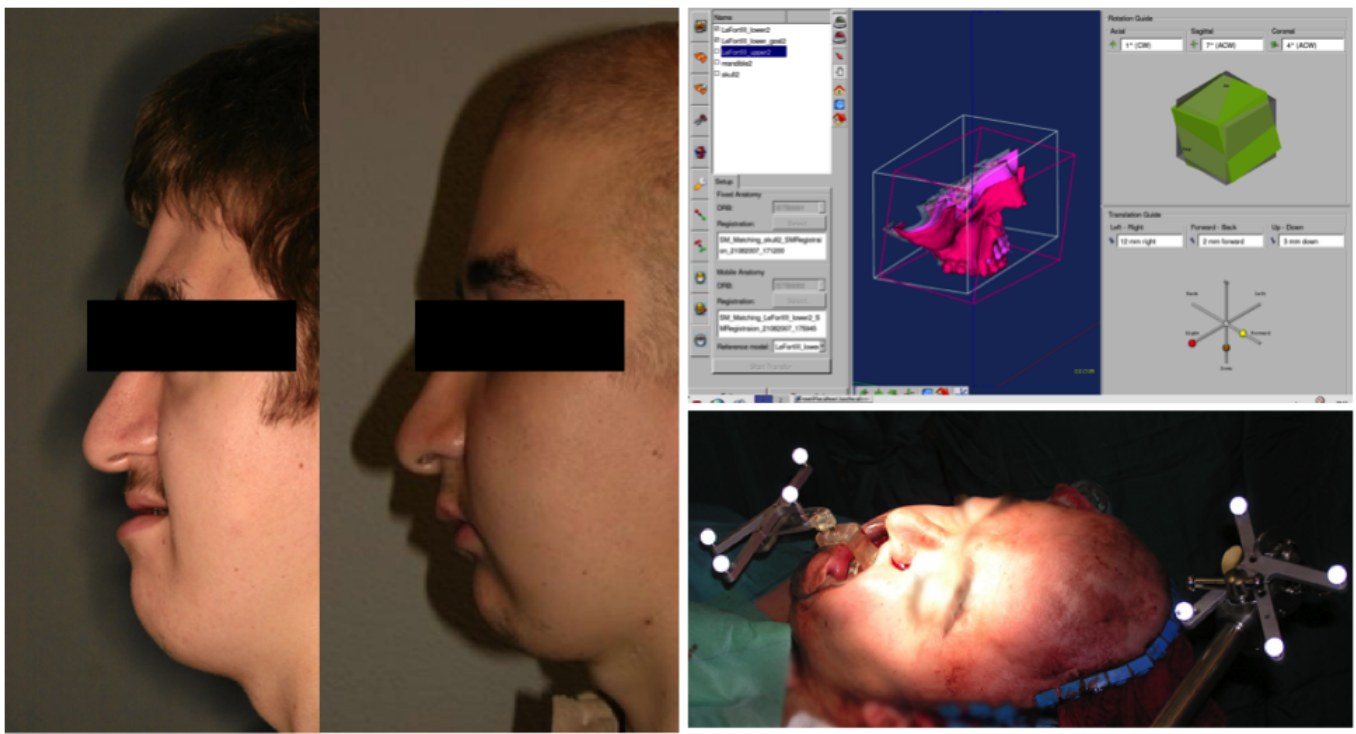

Figure 7: Pre- and post-op soft-tissue profile of the patient 1 (left), intra-op navigation system used during the surgical intervention (right)

experiment was mainly directed to compare the computational performance of these two approaches. In terms of accuracy, the post-operative CT scan was regarded as the ground truth comparison, presented in next section.

\subsection{Validation with post-operative CT scan data}

Post-operative CT scan of the patient was used to compare simulation results in different tissue models: homogeneous and transversely isotropic elasticity. In addition, we evaluated the simulation results in the presence of sliding contact. To assess the accuracy of the simulation results, the distance between each simulation and post-operative result was measured based on corresponding points. Instead of using only closest point matching for defining point correspondence, we adopted a combined approach using TPS 
deformation and closest point matching to minimize the errors in largely deformed area. In the meantime, we used fourteen facial anatomical landmarks on skin surface for TPS deformation as shown in Figure 8.

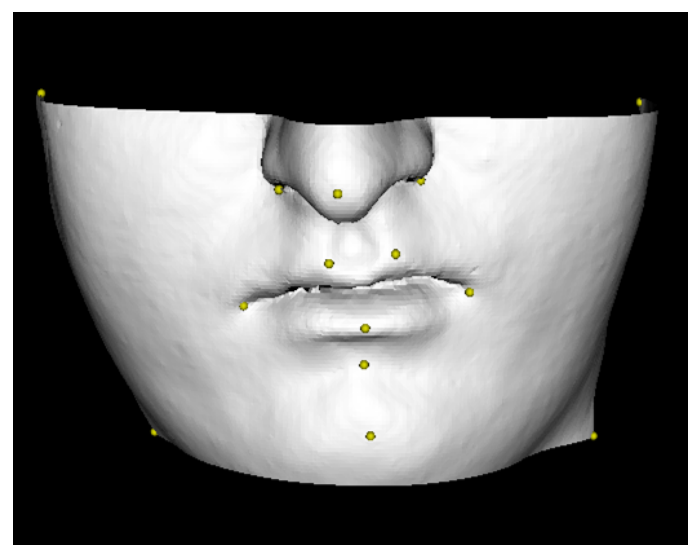

Figure 8: Anatomical landmarks used for defining corresponding points between surfaces

As shown in Figure 9 and Table 3, the mean error is decreased by incorporating transversely isotropy of muscles and sliding contact. These improvements can also be seen qualitatively as color-coded distance errors as shown in Figure 10, where negative value means that predicted skin surface lies behind the post-operative skin surface. Statistical analysis using Wilcoxon test $(p<0.05)$ showed that there was statistically significant difference between the simulation results.

\subsection{Sensitivity analysis of muscle morphing in simulation result}

In order to measure the sensitivity of morphing muscles in simulation result, we performed three different trials defining skull anatomical landmarks proposed by Phillips and Smuts (1996) as shown in Figure 11. The transversely isotropic MTM simulations were then performed using three different 

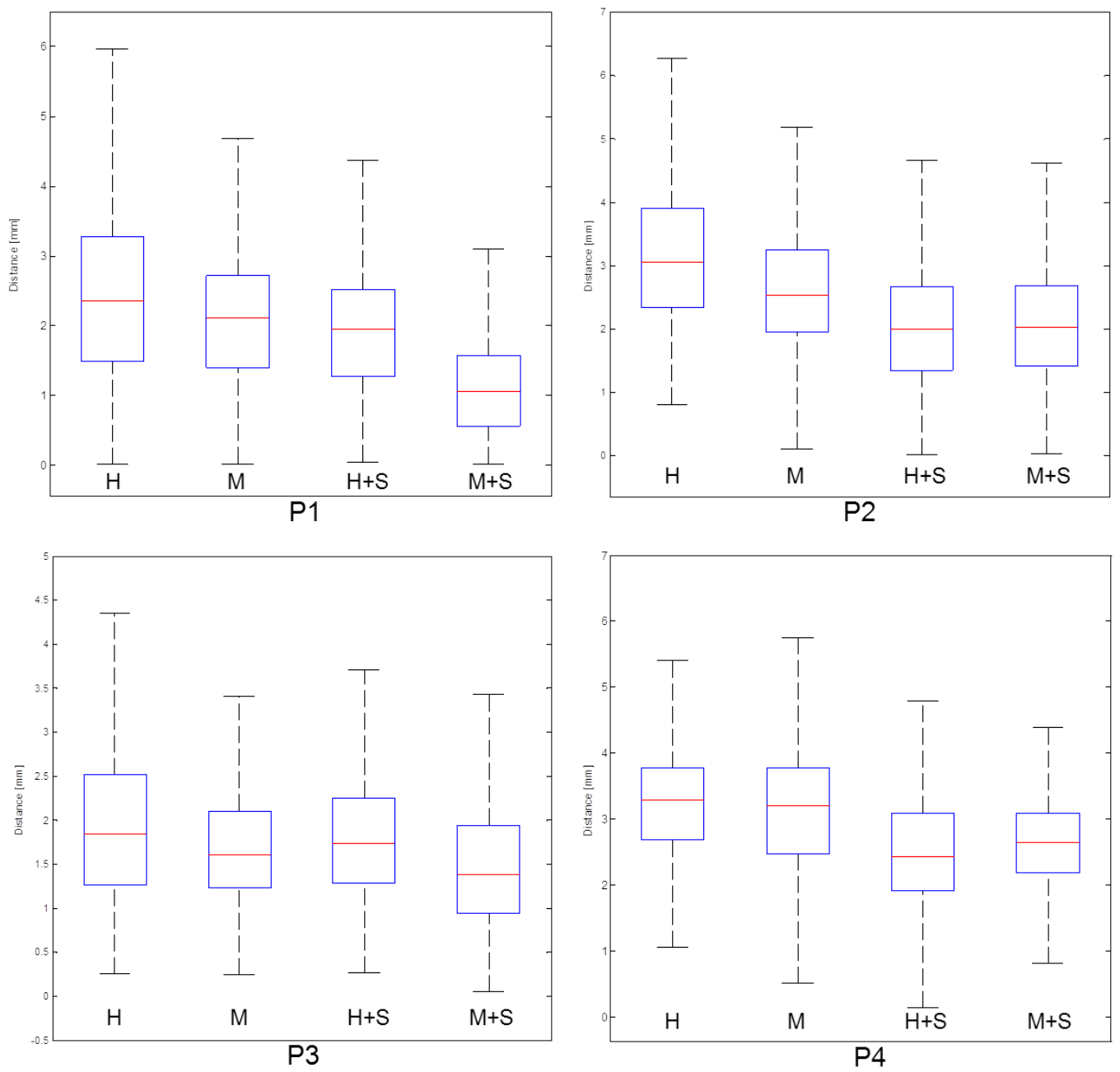

Figure 9: Boxplot of distance errors between simulations and post-operative result for 4 clinical cases (P1,P2,P3,P4); Each graph represents the results with homogeneous $(\mathrm{H})$, muscle template $(\mathrm{M})$, homogeneous + sliding contact $(\mathrm{H}+\mathrm{S})$, and muscle template + sliding contact $(\mathrm{M}+\mathrm{S})$. 
Table 3: Statistical results for 4 patient data.

\begin{tabular}{cccccc}
\hline & & Average Error & Standard Deviation & 90th Percentile & 95th Percentile \\
\hline \multirow{2}{*}{$\mathrm{P} 1$} & $\mathrm{H}$ & 2.50 & 1.21 & 4.10 & 4.40 \\
& $\mathrm{M}$ & 2.21 & 1.23 & 3.39 & 4.16 \\
& $\mathrm{H}+\mathrm{S}$ & 2.07 & 1.24 & 3.68 & 4.39 \\
& $\mathrm{M}+\mathrm{S}$ & 1.21 & 0.92 & 2.15 & 2.90 \\
\hline \multirow{4}{*}{$\mathrm{P} 2$} & $\mathrm{H}$ & 3.25 & 1.27 & 5.13 & 5.85 \\
& $\mathrm{M}+\mathrm{S}$ & 2.68 & 1.07 & 4.00 & 4.65 \\
& $\mathrm{M}+\mathrm{S}$ & 2.11 & 1.06 & 3.42 & 4.06 \\
& $\mathrm{H}$ & 2.03 & 0.98 & 3.42 & 4.04 \\
\hline \multirow{2}{*}{$\mathrm{P} 3$} & $\mathrm{M}$ & 1.80 & 2.41 & 3.01 & 3.38 \\
& $\mathrm{H}+\mathrm{S}$ & 1.75 & 2.36 & 2.61 & 2.87 \\
& $\mathrm{M}+\mathrm{S}$ & 1.58 & 0.71 & 2.61 & 2.78 \\
\hline \multirow{4}{*}{$\mathrm{P} 4$} & $\mathrm{H}$ & 3.23 & 1.90 & 2.58 & 2.84 \\
& $\mathrm{M}$ & 3.10 & 0.83 & 4.25 & 4.48 \\
& $\mathrm{H}+\mathrm{S}$ & 2.43 & 0.99 & 4.27 & 4.51 \\
& $\mathrm{M}+\mathrm{S}$ & 2.56 & 0.84 & 3.46 & 3.67 \\
\hline
\end{tabular}




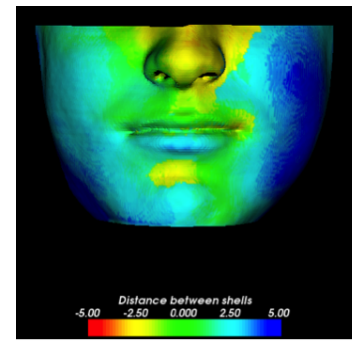

$\mathrm{H}$

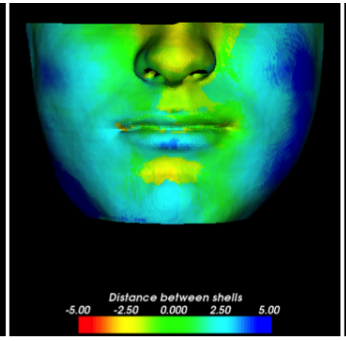

M

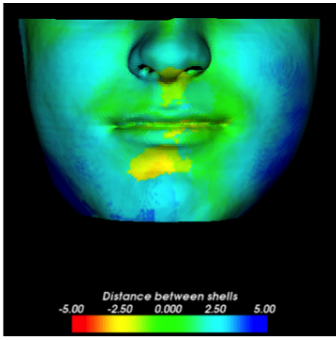

$\mathrm{H}+\mathrm{S}$

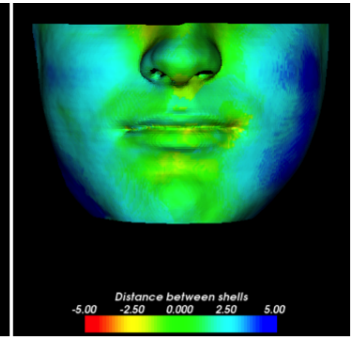

$\mathrm{M}+\mathrm{S}$

Figure 10: Example of color-mapped distance map between simulations for patient 3; Colormap starts from red to blue in the range of $[-5 \mathrm{~mm}, 5 \mathrm{~mm}]$

morphed muscle models. The average deviation in morphed masseter muscles was $2.29 \mathrm{~mm}$ for template muscle I and $1.84 \mathrm{~mm}$ for template muscle II, while the average deviation in simulation results was $0.49 \mathrm{~mm}$ for template muscle I and $0.46 \mathrm{~mm}$ for template muscle II. This highlights the low sensitivity of simulations results in comparison with errors introduced during muscle template morphing procedure.

\section{Discussion and Conclusion}

In this study, we developed a fast and patient-specific soft-tissue simulation method for CMF surgery, with emphasis on its integration into the clinical workflow. A template-based facial muscle morphing technique was proposed to minimize the efforts of segmenting muscle structures without requiring additional imaging modality. In order to improve simulation accuracy, transversely isotropic MTM was applied to realize the directional property of muscle, which was obtained by extracting geometric features. Additionally, sliding contact around teeth and mucosa area was introduced to realize del- 

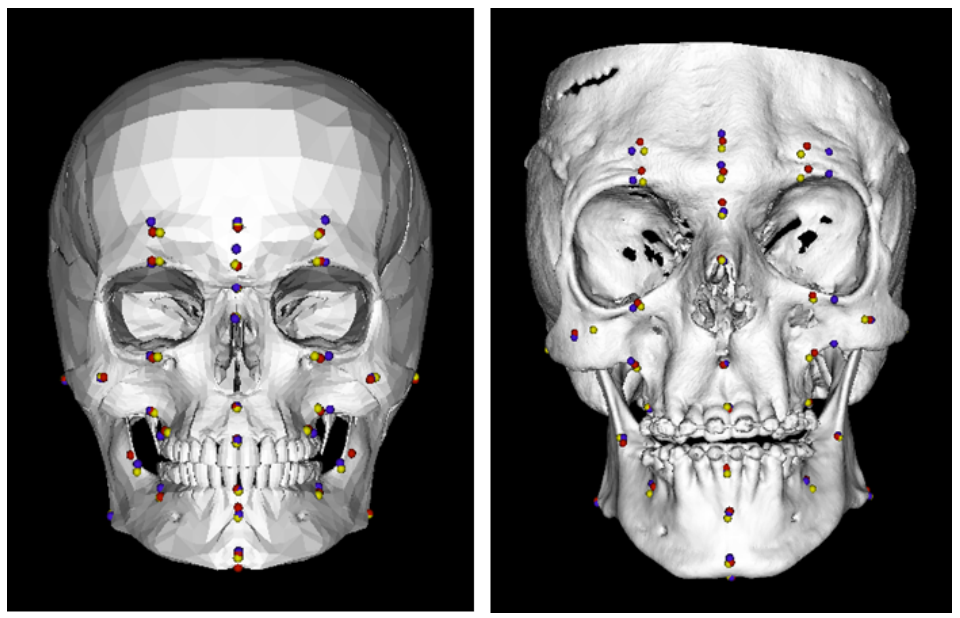

Figure 11: Variability of definition in anatomical landmarks with three independent trials. Each color depicts three different trials.

icate soft-tissue variation in these error-sensitive regions. The method was validated with four clinical cases with post-operative CT scans. The results confirmed that improvements are achieved by considering template-based facial muscles and sliding contact.

As future work, the validity of morphing template models to patientspecific anatomy needs to be further investigated. It is known that significant amount of variability in muscle configurations between individuals is present (Pessa et al., 1998). This aspect should be investigated along with different types of CMF surgery, in order to plausibly determine the importance of such variability. Another limitation of the current study is the linear MTM model utilized, which is insufficient for larger deformations. Furthermore, due to the current lack of information regarding facial tissue characterization, isotropic Poisson ratio for muscle was assumed. Additionally more 
strict control on post-operative scanning will be required to avoid undesirable effects caused by physiological changes in-between pre- and post-operative scans, as well as patient jaw position during scanning. In this sense, combining post-operative surface scanning and navigated operation could replace the need for post-operative CT scanning for evaluation study.

\section{Acknowledgments}

The authors would like to thank the anonymous reviewers for their comments, which helped to improve the quality of this paper. This work was funded by the Swiss National Center of Competence in Research "Computer Aided and Image Guided Medical Interventions (Co-Me)", and the AO/ASIF Foundation, Davos, Switzerland.

\section{References}

Besl, P., McKay, H., 1992. A method for registration of 3-d shapes. Pattern Analysis and Machine Intelligence, IEEE Transactions on 14, 239-256.

Bookstein, F., 1989. Principal warps: thin-plate splines and the decomposition of deformations. Pattern Analysis and Machine Intelligence, IEEE Transactions on $11,567-585$.

Chabanas, M., Luboz, V., Payan, Y., 2003. Patient specific finite element model of the face soft tissues for computer-assisted maxillofacial surgery. Med Image Anal 7, 131-151.

Chapuis, J., Schramm, A., Pappas, I., Hallermann, W., Schwenzer-Zimmerer, K., Langlotz, F., Caversaccio, M., 2007. A new system for computer-aided 
preoperative planning and intraoperative navigation during corrective jaw surgery. Information Technology in Biomedicine, IEEE Transactions on 11, 274-287.

Cotin, S., Delingette, H., Ayache, N., 2000. A hybrid elastic model for realtime cutting, deformations, and force feedback for surgery training and simulation. Vis Comput 16, 437-452.

Duck, F.A., 1990. Physical properties of tissue: a comprehensive reference book, 346 .

Gladilin, E., 2003. Biomechanical modeling of soft tissue and facial expressions for craniofacial surgery planning. Free University Berlin .

Hallermann, W., Olsen, S., Bardyn, T., Taghizadeh, F., Banic, A., Iizuka, T., 2006. A new method for computer-aided operation planning for extensive mandibular reconstruction. Plast Reconstr Surg 117, 2431-7.

Keeve, E., Girod, S., Kikinis, R., Girod, B., 1998. Deformable modeling of facial tissue for craniofacial surgery simulation. Computer Aided Surgery $3,228-238$.

Kim, H., Jürgens, P., Nolte, L.P., Reyes, M., 2010. Anatomically-driven soft-tissue simulation strategy for cranio-maxillofacial surgery using facial muscle template model. Med Image Comput Comput Assist Interv (accepted) .

Koch, R., Gross, M., Carls, F., von Büren, D., Fankhauser, G., Parish, Y., 1996. Simulating facial surgery using finite element models. Proceedings 
of the 23rd annual conference on Computer graphics and interactive techniques, 428 .

Lee, Y., Terzopoulos, D., Waters, K., 1995. Realistic modeling for facial animation. Proceedings of the 22nd annual conference on Computer graphics and interactive techniques, ,55-62.

Lorensen, W., Cline, H., 1987. Marching cubes: A high resolution 3d surface construction algorithm. Proceedings of the 14th annual conference on Computer graphics and interactive techniques, 169.

Mollemans, W., Schutyser, F., Nadjmi, N., Maes, F., Suetens, P., 2006. Parameter optimisation of a linear tetrahedral mass tensor model for a maxillofacial soft tissue simulator. Biomedical Simulation, 159-168.

Mollemans, W., Schutyser, F., Nadjmi, N., Maes, F., Suetens, P., 2007. Predicting soft tissue deformations for a maxillofacial surgery planning system: From computational strategies to a complete clinical validation. Medical Image Analysis 11, 282-301.

Pessa, J.E., Zadoo, V.P., Adrian, E.K., Yuan, C.H., Aydelotte, J., Garza, J.R., 1998. Variability of the midfacial muscles: analysis of 50 hemifacial cadaver dissections. Plast Reconstr Surg 102, 1888-93.

Phillips, V., Smuts, N., 1996. Facial reconstruction: utilization of computerized tomography to measure facial tissue thickness in a mixed racial population. Forensic science international 83, 51-59.

Picinbono, G., Delingette, H., Ayache, N., 2003. Non-linear anisotropic elasticity for real-time surgery simulation. Graphical Models 65, 305-321. 
Roose, L., Maerteleire, W.D., Mollemans, W., Maes, F., Suetens, P., 2006. Simulation of soft-tissue deformations for breast augmentation planning. Biomedical Simulation, 197-205.

Sarti, A., Gori, R., Lamberti, C., 1999. A physically based model to simulate maxillo-facial surgery from $3 \mathrm{~d}$ ct images. Future Generations in Computer Systems 15, 217-222.

Smith, D., Oliker, A., Carter, C., Kirov, M., McCarthy, J., 2007. A virtual reality atlas of craniofacial anatomy. Plastic and reconstructive surgery $120,1641$.

Terzopoulos, D., Waters, K., 1990. Physically-based facial modeling, analysis, and animation. Journal of visualization and Computer Animation 1, 7380.

Teschner, M., Girod, S., Girod, B., 2000. Direct computation of nonlinear soft-tissue deformation. Proc. Vision, Modeling, Visualization VMV'00, 383-390.

Uesu, D., Bavoil, L., Fleishman, S., Shepherd, J., Silva, C., 2005. Simplification of unstructured tetrahedral meshes by point sampling. Volume Graphics, 2005. Fourth International Workshop on , 157- 238.

Zachow, S., Gladiline, E., Hege, H., Deuflhard, P., 2000. Finite-element simulation of soft tissue deformation. Proc. CARS 2000 , 23-28.

Zachow, S., Hierl, T., Erdmann, B., 2004. A quantitative evaluation of 3d soft tissue prediction in maxillofacial surgery planning. Proc. 3. Jahresta- 
gung der Deutschen Gesellschaft fur Computer-und Roboter-assistierte Chirurgie eV, Munchen . 